PROCEEDINGS OF THE

AMERICAN MATHEMATICAL SOCIETY

Volume 127, Number 4, April 1999, Pages 993-1002

S 0002-9939(99)05169-2

\title{
SUCCESSIONAL STABILITY OF VECTOR FIELDS IN DIMENSION THREE
}

\author{
SEBASTIAN J. SCHREIBER
}

(Communicated by Hal L. Smith)

\begin{abstract}
A topological invariant, the community transition graph, is introduced for dissipative vector fields that preserve the skeleton of the positive orthant. A vector field is defined to be successionally stable if it lies in an open set of vector fields with the same community transition graph. In dimension three, it is shown that vector fields for which the origin is a connected component of the chain recurrent set can be approximated in the $C^{1}$ Whitney topology by a successionally stable vector field.
\end{abstract}

\section{INTRODUCTION}

Ever since the founding work of Lotka and Volterra, theoretical ecologists and mathematicians have studied dissipative systems of the form

$$
\frac{d x}{d t}=f(x)
$$

where $x=\left(x_{1}, \ldots, x_{n}\right)$ is a vector of species' densities, $f=\left(f_{1}, \ldots, f_{n}\right)$ is the vector of species' growth rates and $f_{i}(x)=0$ whenever $x_{i}=0$. In studying (1), a lot of attention has revolved around finding conditions that result in coexistence or exclusion. Coexistence refers to the long term survival of a collection of species and has been equated with the existence of a local $[1,9,20]$ or global $[3,4,11,14,26,27]$ attractor that lies in the interior of the positive orthant. In contrast, exclusion refers to the extinction of a subset of species and has been equated with the existence of a local or global attractor that lies in the boundary of the positive orthant $[1,12,13,29,31]$. By studying both of these processes for all subsets of species in (1), theoretical ecologists have begun to examine what rules govern the assembly of ecological communities $[5,7,19,24]$. Inspired by this work, we introduce a topological invariant, the community-transition graph, for (1). This invariant is a graph with directed and labeled edges whose vertices represent communities (permanent/uniformly persistent subsystems of (1)) and whose edges represent transitions from one community to another community due to invasions by other species.

Received by the editors January 28, 1997.

1991 Mathematics Subject Classification. Primary 34D30, 58F12; Secondary 92D40, 92D25.

Key words and phrases. Generic properties of vector fields, ecological succession, population dynamics.

Part of this research was completed during a postdoctoral fellowship sponsored by Andrew P. Gutierrez at the University of California, Berkeley. For his support and encouragement, the author is grateful. 
Since in the natural world only robust phenomena are observable, we define $f$ to be successionally stable if its community transition graph remains unaltered following sufficiently small perturbations of $f$. The main theorem of this article shows that successionally stable vector fields are dense amongst "reasonable" vector fields in dimension three where by "reasonable" we mean the origin is a connected component of the chain recurrent set. This condition is met by most models of population growth.

\section{Preliminaries and statement of the main theorem}

Let $\mathbf{R}_{+}^{n}$ denote the closed positive orthant of $\mathbf{R}^{n}$. For any subset $S \subseteq\{1, \ldots, n\}$ define $\mathbf{R}^{S}=\left\{\left(x_{1}, \ldots, x_{n}\right) \in \mathbf{R}^{n}: x_{i}=0 \forall i \notin S\right\}$ and $\mathbf{R}_{+}^{S}=\mathbf{R}^{S} \cap \mathbf{R}_{+}^{n}$. If $|S|=k$, then $\mathbf{R}_{+}^{S}$ is a $k$-dimensional face of $\mathbf{R}_{+}^{n}$. The $k$-skeleton of $\mathbf{R}_{+}^{n}$ is the union of the $k$-dimensional faces of $\mathbf{R}_{+}^{n}$. Given $S \subseteq\{1, \ldots, n\}$ and $A \subseteq \mathbf{R}_{+}^{S}$, we let $\partial A, \bar{A}$ and int $A$ denote the boundary, closure and interior of $A$ relative to the topology of $\mathbf{R}_{+}^{S}$.

We recall several definitions from dynamical systems theory. Given a $C^{1}$ vector field, $f: \mathbf{R}_{+}^{n} \rightarrow \mathbf{R}^{n}$, that generates a global flow, $\phi: \mathbf{R} \times \mathbf{R}_{+}^{n} \rightarrow \mathbf{R}_{+}^{n}$, define $\phi_{t}(x)=\phi(t, x)$. A set $K \subset \mathbf{R}_{+}^{n}$ is called $f$ invariant if $\phi_{t}(K)=K$ for all $t \in \mathbf{R}$. An $f$ invariant set $K$ is called an attractor for $f$ provided there exists an open neighborhood $U$ of $K$ such that $\phi_{t}(\bar{U}) \subset \operatorname{int} U$ for sufficiently large $t$ and $K=$ $\bigcap_{t>0} \phi_{t}(U)$. An invariant set $K$ is isolated if there exists a neighborhood $U$ of $K$ such that $K$ is the maximal invariant set in $U$. In this case, $U$ is called an isolating neighborhood of $K$. Given $x \in \mathbf{R}_{+}^{n}$, the omega limit set of $x, \omega(f, x)$, is the collection of points $y \in \mathbf{R}_{+}^{n}$ such that there exists an increasing sequence $t_{k} \uparrow \infty$ satisfying $\phi_{t_{k}}(x) \rightarrow y$ as $k \rightarrow \infty$. Given $x \in \mathbf{R}_{+}^{n}$, the alpha limit set of $x, \alpha(f, x)$, is the collection of points $y \in \mathbf{R}_{+}^{n}$ such that there exists a decreasing sequence $t_{k} \downarrow-\infty$ satisfying $\phi_{t_{k}}(x) \rightarrow y$ as $k \rightarrow \infty$. The stable set and unstable set of an isolated invariant set $K$ are defined by

$$
W^{s}(f, K)=\left\{x \in \mathbf{R}_{+}^{n}: \omega(f, x) \neq \emptyset \text { and } \omega(f, x) \subseteq K\right\}
$$

and

$$
W^{u}(f, K)=\left\{x \in \mathbf{R}_{+}^{n}: \alpha(f, x) \neq \emptyset \text { and } \alpha(f, x) \subseteq K\right\} .
$$

A vector field $f: \mathbf{R}_{+}^{n} \rightarrow \mathbf{R}^{n}$ is dissipative if there exists a compact attractor $K \subset \mathbf{R}_{+}^{n}$ such that $\omega(f, x) \subseteq K$ for all $x \in \mathbf{R}_{+}^{n}$. A point $x \in K$ is called chain recurrent for $f$ restricted to $K$ provided that for every $\epsilon>0$ and $T>0$ there exist points in $K, x=x_{0}, x_{1}, \ldots, x_{k}=x$, and real numbers, $t_{0}, \ldots, t_{k-1}$, greater than $T$ such that $d\left(\phi\left(t_{i}, x_{i}\right), x_{i+1}\right)<\epsilon$ for all $0 \leq i \leq k-1$. Let $\mathcal{R}(f, K)$ denote the set of chain recurrent points for $f$ restricted to $K$. Let $\mathcal{R}(f)$ refer to the chain recurrent set of $f$ on its entire domain.

At this point, we are ready to introduce the definitions necessary to state our main result.

Definition 1. Let $\mathcal{P}_{n}$ be the set of $C^{1}$ dissipative vector fields $f: \mathbf{R}_{+}^{n} \rightarrow \mathbf{R}^{n}$ such that $f_{i}(x)=0$ whenever $x_{i}=0$.

We view $\mathcal{P}_{n}$ as the space of all possible descriptions of $n$ interacting species and endow it with the $C^{1}$ Whitney topology (uniform convergence on compact sets).

Given $f \in \mathcal{P}_{n}, f$ is called permanent $[11,27]$ or, equivalently, uniformly persistent $[3,4]$ provided that there exists a compact attractor $K \subset \operatorname{int} \mathbf{R}_{+}^{n}$ such that 

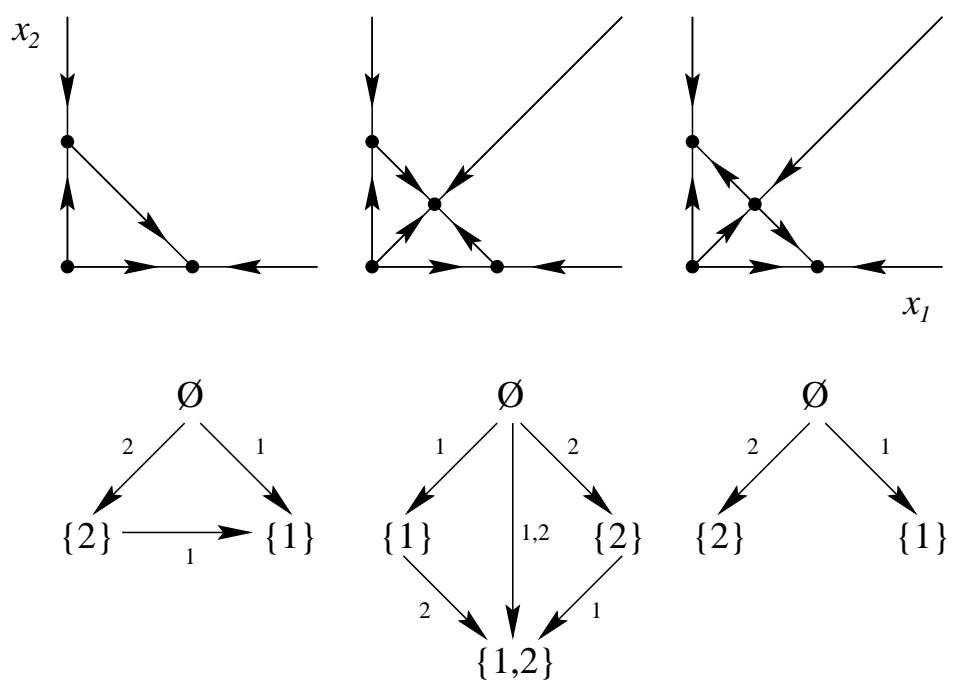

FIgURE 1. The phase diagrams and corresponding community transition graphs for the Lotka-Volterra equations of two competing species

$\omega(f, x) \subseteq K$ for all $x \in \operatorname{int} \mathbf{R}_{+}^{n}$. Given $S \subseteq\{1, \ldots, n\}$, let $f_{S}$ denote $f$ restricted to $\mathbf{R}_{+}^{S}$.

Definition 2. $S \subseteq\{1, \ldots, n\}$ is called a community for (1) if $f_{S}$ is uniformly persistent. Equivalently, there exists a compact attractor $K \subset \operatorname{int} \mathbf{R}_{+}^{S}$ such that $\omega(f, x) \subseteq K$ for all $x \in \operatorname{int} \mathbf{R}_{+}^{S}$.

Definition 3. Assume $S$ and $T$ are distinct communities for (1) and that $I \subseteq$ $\{1, \ldots, n\} \backslash S$. There is a transition from $S$ to $T$ due to an invasion by $I$ if $\omega(f, x) \subseteq$ $\mathcal{R}\left(f_{T}\right) \cap \operatorname{int} \mathbf{R}_{+}^{T}$ for all $x \in \operatorname{int} \mathbf{R}_{+}^{S \cup I}$. When this occurs, we write $S \stackrel{I}{\rightarrow} T$.

Definition 4. Given $f \in \mathcal{P}_{n}$, the community transition graph of $f, \Gamma(f)$, is a directed graph with labeled edges: the vertices represent communities of $f$ and the edges between $S$ and $T$ represent all possible transitions from $S$ to $T$.

Definitions 2, 3 and 4 essentially are rigorous restatements of definitions found in the ecological literature $[18,19]$. The main difference is that our definitions account for multiple species invasions (i.e., $|I|>1$ ) while the ecological literature thus far only considers single species invasions. In Figure 1, we illustrate these definitions with the Lotka-Volterra equations for two competing species.

The community transition graph defines an equivalence relation on $\mathcal{P}_{n}$.

Definition 5. Assume $f, g \in \mathcal{P}_{n}$. $f$ is successionally equivalent to $g$ provided there exists a permutation $\sigma$ of the coordinates of $\mathbf{R}_{+}^{n}$ such that $\Gamma\left(\sigma \circ f \circ \sigma^{-1}\right)=\Gamma(g)$.

Using this equivalence relationship, we make the following

Definition 6. $f \in \mathcal{P}_{n}$ is successionally stable if it is contained in an open set of successionally equivalent vector fields. 
Our main result is

Theorem 1. Let $f \in \mathcal{P}_{3}$ be such that the origin is a connected component of $\mathcal{R}\left(f, \partial \mathbf{R}_{+}^{3}\right)$. Then every neighborhood $\mathcal{N} \subseteq \mathcal{P}_{3}$ of $f$ contains successionally stable vector fields.

Remark 1 . The condition that the origin is a connected component of $\mathcal{R}\left(f, \partial \mathbf{R}_{+}^{3}\right)$ is met by most ecological models. For instance, if $f \in \mathcal{P}_{3}$ is a vector field such that $f$ has at most one equilibrium on the interior of any 1-dimensional face and such that the equilibria on the 1-skeleton are hyperbolic, then this condition is met.

\section{Community Robustness}

The approximation of a vector field by a successionally stable vector field depends on a simpler problem. This problem involves the notion of community robustness of a vector field which, roughly stated, means that no communities are created or destroyed by small perturbations of the vector field.

Definition 7. $f \in \mathcal{P}_{n}$ has robust communities provided that there is a neighborhood $\mathcal{N} \subseteq \mathcal{P}_{n}$ of $f$ such that for every $S \subseteq\{1, \ldots, n\}$ either $S$ is a community for all $h \in \mathcal{N}$ or $S$ is not a community for all $h \in \mathcal{N}$.

To approximate a vector field by a successional stable vector field, we need to address the following question:

Question 1. Given $f \in \mathcal{P}_{n}$, can we approximate $f$ by a vector field with robust communities?

Thus far, this question has received little study (see [14] for discussion). An affirmative answer to this question for $n \leq 2$ is easily proven. In this section we answer the question for "reasonable" vector fields when $n=3$.

Theorem 2. Let $f$ be a vector field in $\mathcal{P}_{3}$ such that the origin is a connected component of $\mathcal{R}\left(f, \partial \mathbf{R}_{+}^{3}\right)$. Then every neighborhood $\mathcal{N} \subseteq \mathcal{P}_{3}$ of $f$ contains vector fields with robust communities.

To prove this theorem we use Garay's characterization of uniform persistence in terms of the stable sets of the connected components of $\mathcal{R}\left(f, \partial \mathbf{R}_{+}^{3}\right)[8]$.

Theorem 3 (Garay 1989). Let $f \in \mathcal{P}_{n}$. Suppose every connected component $C$ of $\mathcal{R}\left(f, \partial \mathbf{R}_{+}^{n}\right)$ is an isolated invariant set and $W^{s}(f, C) \cap \operatorname{int} \mathbf{R}_{+}^{n}=\emptyset$. Then $f$ is uniformly persistent.

For our purposes, it is sufficient to understand the chain recurrent set of the boundary flow generically. This is facilitated by the following definition.

Definition 8. A vector field $f \in \mathcal{P}_{n}$ is called Kupka-Smale if

1. Each element of the set of periodic orbits and equilibrium points, $\operatorname{Per}(f)$, is hyperbolic.

2. For any $S \subseteq\{1, \ldots, n\}$ and $\tau_{1}, \tau_{2} \in \operatorname{Per}\left(f_{S}\right)$, all points of non-transversal intersection of $W^{s}\left(f_{S}, \tau_{1}\right)$ and $W^{u}\left(f_{S}, \tau_{2}\right)$ lie in $\partial \mathbf{R}_{+}^{S}$.

Definition 8 differs from the traditional definition of Kupka-Smale vector fields for manifolds without boundary in that it permits non-transversal intersections of stable and unstable manifolds. This difference is necessary as these non-transversal intersections can not be perturbed away within $\mathcal{P}_{n}$. For compact manifolds without 


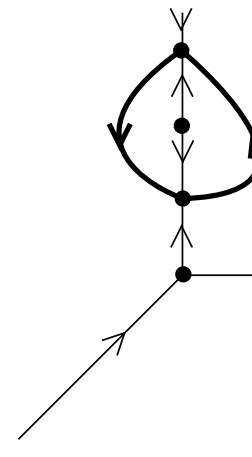

(a)

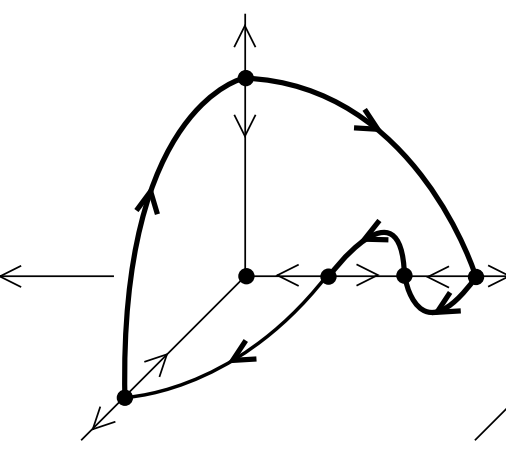

(b)

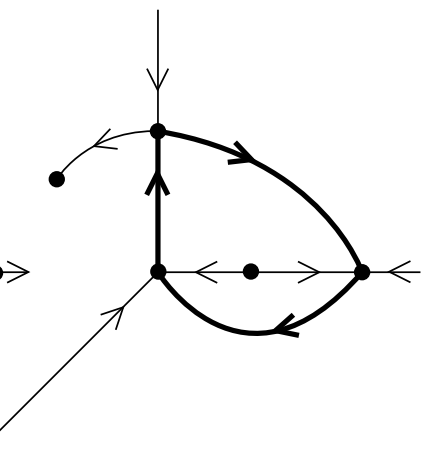

(c)

FiguRE 2. Three types of robust heteroclinic cycles in $\partial \mathbf{R}_{+}^{3}$ : (a) attracting, (b) repelling and (c) semi-stable.

boundary, Kupka [17] and Smale [28] independently proved that the set of KupkaSmale vector fields is residual (i.e. a countable intersection of open and dense sets). Peixoto [23] extended this result to non-compact manifolds without boundary. Since for dissipative vector fields on $\mathbf{R}_{+}^{n}$ the non-compact proof applies to the interior of any face of $\mathbf{R}_{+}^{n}$, it follows that Kupka-Smale vector fields as defined above are residual in $\mathcal{P}_{n}$. Therefore, given $f \in \mathcal{P}_{n}$ we can always approximate it by a KupkaSmale vector field that lies in $\mathcal{P}_{n}$.

To characterize the connected components of the chain recurrent set for a KupkaSmale vector field, recall that a set of $k$ distinct equilibria $\left\{p_{1}, \ldots, p_{k}\right\}$ defines a heteroclinic cycle $\gamma$ if $k \geq 2$ and $W^{u}\left(p_{i}\right) \cap W^{s}\left(p_{i+1}\right) \neq \emptyset$ for all $1 \leq i \leq k$ where we identify $p_{k+1}$ with $p_{1}$. When this occurs, we write $\gamma=\bigcup_{i=1}^{k} W^{u}\left(p_{i}\right) \cap W^{s}\left(p_{i+1}\right)$.

Proposition 4. Let $f \in \mathcal{P}_{3}$ be Kupka-Smale. If $C$ is a connected component of $\mathcal{R}\left(f, \partial \mathbf{R}_{+}^{3}\right)$, then one of the following statements apply:

1. $C \in \operatorname{Per}(f)$.

2. $C$ is a heteroclinic cycle consisting of $k$ distinct equilibria $p_{1} \in \operatorname{int} \mathbf{R}_{+}^{\{\sigma(1)\}}, \ldots$, $p_{k} \in \operatorname{int} \mathbf{R}^{\{\sigma(k)\}}$ where $\sigma(i) \in\{1,2,3\}$. Furthermore, this heteroclinic cycle is either attracting (i.e $\frac{\partial f_{\sigma(i)}}{\partial x_{\sigma(i)}}\left(p_{i}\right)<0$ for all $1 \leq i \leq k$ ) or repelling (i.e. $\frac{\partial f_{\sigma(i)}}{\partial x_{\sigma(i)}}\left(p_{i}\right)>0$ for all $\left.1 \leq i \leq k\right)$ for $f$ restricted to $\partial \mathbf{R}_{+}^{3}$.

3. $C$ includes a heteroclinic orbit $\gamma$ that passes through the origin.

Several of the possibilities are illustrated in Figure 2. Notice that when the chain component $C$ contains a heteroclinic cycle that passes through the origin, then this heteroclinic cycle can be stable, unstable or semi-stable for $f$ restricted to $\partial \mathbf{R}_{+}^{3}$.

Proof. Let $f_{\partial}$ denote $f$ restricted to $\partial \mathbf{R}_{+}^{3}$. Since $f$ is Kupka- Smale and $\partial \mathbf{R}_{+}^{3}$ is two dimensional, a standard argument shows that $\operatorname{Per}\left(f_{\partial}\right)$ is finite (see, for example, Chapter 4.2 in [22]). Let $A$ be the set of equilibria in $\partial \mathbf{R}_{+}^{3}$ that are saddles for $f_{\partial}$. The set $A \backslash\{0\}$ consists of three types of equilibria: those in the interior of a 1 -face $\mathbf{R}_{+}^{\{i\}}$ that are stable for $f_{\{i\}}$, those in the interior of a 1-face $\mathbf{R}_{+}^{\{i\}}$ that are unstable for $f_{\{i\}}$, and those that lie in the interior of a 2 -face. We call these sets $A_{s}, A_{u}$ and $A_{o}$, respectively. Let $B=\operatorname{Per}\left(f_{\partial}\right) \backslash A$. Transversality of the stable 
and unstable manifolds in the interior of the 2-faces implies that no 2 -face contains a heteroclinic cycle. This observation and the Poincaré-Bendixson theorem imply that $\partial \mathbf{R}_{+}^{3}=W^{s}\left(f_{\partial}, A\right) \cup W^{s}\left(f_{\partial}, B\right)$.

Let $C$ be a connected component of $\mathcal{R}\left(f_{\partial}\right)$. Suppose $C \notin \operatorname{Per}(f)$. The PoincaréBendixson theorem implies that $C$ contains a heteroclinic cycle $\gamma \subseteq W^{s}\left(f_{\partial}, A\right)$. Suppose $\gamma$ is defined by $k$ distinct equilibria $\left\{p_{1}, \ldots, p_{k}\right\} \subset A \backslash\{0\}$. Transversality of the stable and unstable manifolds in the interior of the 2-faces implies that $\left\{p_{1}, \ldots, p_{k}\right\} \subseteq A_{s} \cup A_{u}$. Suppose $p_{1} \in A_{s}$. Since $f$ is Kupka-Smale and $\gamma$ is a heteroclinic orbit, $W^{u}\left(f_{\partial}, p_{1}\right)$ and $W^{s}\left(f_{\partial}, p_{2}\right)$ intersect non-trivially and transversely in the interior of a 2-face. As $W^{u}\left(f, A_{s}\right) \cap \partial \mathbf{R}_{+}^{3}$ and $W^{s}\left(f, A_{u}\right) \cap \partial \mathbf{R}_{+}^{3}$ cannot intersect non-trivially and transversely in the interior of a 2 -face, $p_{2}$ must lie in $A_{s}$. Induction implies that $\left\{p_{1}, \ldots, p_{k}\right\} \subseteq A_{s}$. In this case, $\gamma$ is an attractor for $f_{\partial}$ and $\gamma=C$. On the other hand, suppose that $p_{1} \in A_{u}$. As $W^{s}\left(f_{\partial}, p_{1}\right)$ and $W^{u}\left(f_{\partial}, p_{k}\right)$ intersect non-trivially and transversely in the interior of a 2 -face, $p_{k}$ must lie in $A_{u}$. Induction implies $\left\{p_{1}, \ldots, p_{k}\right\} \subseteq A_{u}$. In this case, $\gamma$ is a repellor for $f_{\partial}$ and $\gamma=C$.

While the stable manifold theorem provides a local description of the stable sets of hyperbolic periodic orbits and equilibria, we need some additional definitions to understand the stable sets of heteroclinic cycles. Assume $\gamma \subset \partial \mathbf{R}_{+}^{3}$ is a heteroclinic cycle for a Kupka-Smale vector field and is determined by the equilibria $p_{1} \in$ $\operatorname{int} \mathbf{R}^{\{\sigma(1)\}}, \ldots, p_{k} \in \operatorname{int} \mathbf{R}^{\{\sigma(k)\}}$. The geometry of this heteroclinic cycle allows us to divide the eigenvalues of the linearized flow at an equilibrium $p_{i}$ into three classes. There are the radial eigenvalues $\frac{\partial f_{\sigma(i)}}{\partial x_{\sigma(i)}}\left(p_{i}\right)$ that determine whether the heteroclinic cycle is attracting or repelling for the flow restricted to $\partial \mathbf{R}_{+}^{3}$. Along the heteroclinic cycle, there is the expanding eigenvalue $e_{i}$ (i.e, the largest eigenvalue of $\operatorname{Df}\left(p_{i}\right)$ corresponding to an eigenvector $v \notin \mathbf{R}^{\{\sigma(i)\}}$ ) and the contracting eigenvalue $c_{i}$ (i.e., the largest eigenvalue of $-D f\left(p_{i}\right)$ corresponding to an eigenvector $\left.v \notin \mathbf{R}^{\{\sigma(i)\}}\right)$. With these definitions in place, we get the following lemma which is based upon results of $[2,4,10,16]$.

Lemma 5. Let $f \in \mathcal{P}_{3}$ be a Kupka-Smale vector field. Suppose that $f$ restricted to $\partial \mathbf{R}_{+}^{3}$ has an attracting or repelling heteroclinic cycle $\gamma$ determined by the equilibria, $p_{1} \in \operatorname{int} \mathbf{R}_{+}^{\{\sigma(1)\}}, \ldots, p_{k} \in \operatorname{int} \mathbf{R}_{+}^{\{\sigma(k)\}}$.

1. If $\prod_{i=1}^{k} e_{i}>\prod_{i=1}^{k} c_{i}$, then $\gamma$ is an isolated invariant set and $W^{s}(f, \gamma) \subseteq \partial \mathbf{R}_{+}^{3}$.

2. If $\prod_{i=1}^{k} c_{i}>\prod_{i=1}^{k} e_{i}$, then $\gamma$ is an isolated invariant set and $W^{s}(f, \gamma) \cap$ $\operatorname{int} \mathbf{R}_{+}^{3} \neq \emptyset$.

Remark 2. It is exactly here that a proof for density of successional stability is technically obstructed. In particular, we know of no algebraic criterion that provides information about the stable sets for heteroclinic cycles of the type illustrated in Figure 2c.

Proof. Let $f_{\partial}$ denote $f$ restricted to $\partial \mathbf{R}_{+}^{3}$. When $\gamma$ is an attractor for $f_{\partial}$, the assertions of this lemma are well-known and have been proven using either average Lyapunov functions [10] or Poincaré sections $[2,16]$. In fact these authors prove that if $\prod_{i=1}^{k} c_{i}>\prod_{i=1}^{k} e_{i}$, then $\gamma$ is an attractor for $f$.

To deal with the case when $\gamma$ is a repellor for $f_{\partial}$, it suffices to investigate the behavior of the flow generated by $-f$. For $-f_{\partial}, \gamma$ is attracting. Furthermore if the $c_{i}\left(e_{i}\right)$ are the contracting (expanding) eigenvalues for $f$, then the $e_{i}\left(c_{i}\right)$ are 
the contracting (expanding) eigenvalues for $-f$. Suppose $\prod_{i=1}^{k} e_{i}>\prod_{i=1}^{k} c_{i}$; then $\gamma$ is an attractor for $-f$. Hence $W^{s}(f, \gamma)=W^{u}(-f, \gamma)=\gamma$. Now suppose that $\prod_{i=1}^{k} c_{i}>\prod_{i=1}^{k} e_{i}$; then $W^{s}(-f, \gamma) \subseteq \partial \mathbf{R}_{+}^{3}$. We want to show that $W^{s}(f, \gamma) \cap$ int $\mathbf{R}_{+}^{3}=W^{u}(-f, \gamma) \cap \operatorname{int} \mathbf{R}_{+}^{3} \neq \emptyset$. This can be accomplished by modifying an argument of Butler and Waltman [4, Lemma 2.1] as follows. Since $\gamma$ is an isolated invariant set for $-f$, let $V \subset \mathbf{R}_{+}^{3}$ be a compact isolating neighborhood such that $U=V \cap \partial \mathbf{R}_{+}^{3}$ is contained in $W^{s}(-f, \gamma)$. Let $\phi(t, x)$ be the flow generated by $-f$. Define $\phi([0, t), q)=\{\phi(s, q): 0 \leq s<t\}$. Since $W^{s}(-f, U) \subseteq \partial \mathbf{R}_{+}^{3}$ and $V$ is an isolating neighborhood, for each $q \in V \backslash U$, there exists $t(q) \geq 0$ such that $\phi(t(q), q) \in \partial V \backslash U$ and $\phi([0, t(q)), q) \subset V$. Consider a sequence of points $q_{m} \in V \backslash U$ such that $q_{m}$ converge to a point in $\gamma$. Let $m_{k}$ be a subsequence such that $\phi\left(t\left(q_{m_{k}}\right), q_{m_{k}}\right)$ converges to a point $y \in \partial V$. Since $q_{m_{k}}$ converges to a point in $\gamma$, it follows that $t\left(q_{m_{k}}\right) \rightarrow \infty$. Compactness of $V$ and continuity of $\phi$ imply that $\phi((-\infty, 0], y) \subseteq V$. Since $U \subseteq W^{s}(-f, \gamma), y$ must lie in $V \backslash U$. As $V$ is an isolating neighborhood, we get $\alpha(-f, y) \subseteq \gamma$. Hence $W^{u}(-f, \gamma) \cap \operatorname{int} \mathbf{R}_{+}^{3} \neq \emptyset$.

Now, we are ready to prove Theorem 2. Let $f \in \mathcal{P}_{3}$ be such that 0 is a connected component of $\mathcal{R}\left(f, \partial \mathbf{R}_{+}^{3}\right)$. Let $\mathcal{N} \subseteq \mathcal{P}_{3}$ be a neighborhood of $f$. Conley [6] proved there is a continuous Lyapunov function $V: \partial \mathbf{R}_{+}^{3} \rightarrow \mathbf{R}_{+}$such that $V$ is strictly decreasing off of $\mathcal{R}\left(f, \partial \mathbf{R}_{+}^{3}\right)$. Using the smoothing techniques of Wilson [30] (see, e.g., [21, Proposition 6]), $V$ can be made to be $C^{\infty}$ and to satisfy $D V(x) g(x)<0$ for all $x \in \partial \mathbf{R}_{+}^{3} \backslash \mathcal{R}\left(f, \partial \mathbf{R}_{+}^{3}\right)$ and $V^{-1}([0, c])$ is compact for all $c \geq 0$.

Remark 3. The work of Conley applies to flows on compact manifolds. To compactify the flow of $g \in \mathcal{P}_{n}$, let $\beta: \mathbf{R}_{+}^{n} \rightarrow(0,1]$ be a smooth function that approaches 0 sufficiently fast as $|x| \rightarrow \infty$ (e.g., $\beta(x)=\exp \left(-|x|^{2}-|f(x)|^{2}\right)$ ). The flow of $\beta g$ extends to a flow on the one point compactification of $\mathbf{R}_{+}^{n}$. The work of Conley applies to this flow. Since $\beta g$ is just a reparameterization of $g$, any Lyapunov function for $\beta g$ is also a Lyapunov function for $g$.

Since 0 is a connected component of $\mathcal{R}\left(f, \partial \mathbf{R}_{+}^{3}\right)$, there exists an open neighborhood $U \subset \partial \mathbf{R}_{+}^{3}$ of 0 such that $D V(x) f(x)<0$ for all $x \in U \backslash\{0\}$. Our choice of $U$ implies there exists a neighborhood $\mathcal{N}_{0} \subseteq \mathcal{N}$ of $f$ such that $U \cap \mathcal{R}\left(g, \partial \mathbf{R}_{+}^{3}\right)$ is compact for all $g \in \mathcal{N}_{0}$. Since 0 is the only equilibrium for $f$ in $U, f_{i}(x)$ is nonzero for $x \in \bigcup_{i} \operatorname{int} \mathbf{R}_{+}^{\{i\}} \cap U$. Therefore we can choose a Kupka-Smale vector field $g \in \mathcal{N}_{0}$ such that $\operatorname{Per}(g) \cap U \cap\left(\bigcup_{i} \mathbf{R}_{+}^{\{i\}}\right)=\{0\}$ and such that any heteroclinic orbit $\gamma \subset \partial \mathbf{R}_{+}^{3} \backslash\{0\}$ for $g$ satisfies one of the eigenvalue inequalities of Lemma 5. Since $U \cap \mathcal{R}\left(f, \partial \mathbf{R}_{+}^{3}\right)$ is compact and $\operatorname{Per}(g) \cap U \cap\left(\bigcup_{i} \mathbf{R}_{+}^{\{i\}}\right)=\{0\}$, Proposition 4 implies that 0 is connected component of $\mathcal{R}\left(g, \partial \mathbf{R}_{+}^{3}\right)$.

Let $L: \partial \mathbf{R}_{+}^{3} \rightarrow \mathbf{R}_{+}$be a smooth Lyapunov function for $g$ (i.e. $D L(x) g(x)<0$ for all $\left.x \in \partial \mathbf{R}_{+}^{3} \backslash \mathcal{R}\left(g, \partial \mathbf{R}_{+}^{3}\right)\right)$. Let $a_{1}, \ldots, a_{m}$ be distinct non-negative reals such that the connected components of $\mathcal{R}\left(g, \partial \mathbf{R}_{+}^{3}\right)$ are given by $C_{i}(g)=L^{-1}\left(a_{i}\right)$. Choose $\delta>0$ sufficiently small such that the following fact holds:

(F) The sets $U_{i}=L^{-1}\left(\left(a_{i}-\delta, a_{i}+\delta\right)\right)$ are disjoint.

Since $D L(x) g(x)<0$ for all $x \in \partial \mathbf{R}_{+}^{3} \backslash \mathcal{R}\left(g, \partial \mathbf{R}_{+}^{3}\right)$, there exists a neighborhood $\mathcal{N}_{1} \subseteq \mathcal{N}_{0}$ of $g$ such that every $h \in \mathcal{N}_{1}$ satisfies the following property:

(P1) $D L(x) h(x)<0$ for all $x \in \partial \mathbf{R}_{+}^{3} \backslash \bigcup_{i} U_{i}$. 
Hyperbolicity of the elements of $\operatorname{Per}(g)$ implies that there exists a neighborhood $\mathcal{N}_{2} \subseteq \mathcal{N}_{1}$ such that each $h \in \mathcal{N}_{2}$ satisfies (see, e.g., Proposition 3.1 in Chapter 2 of $[22])$

(P2) $h$ restricted to $\partial \mathbf{R}_{+}^{3}$ is Kupka-Smale and $\left|\operatorname{Per}(h) \cap U_{i} \cap \operatorname{int} \mathbf{R}_{+}^{S}\right|=$ $\left|\operatorname{Per}(g) \cap U_{i} \cap \operatorname{int} \mathbf{R}_{+}^{S}\right|$ for all $S \subset\{1,2,3\}$.

For $h \in \mathcal{N}_{2}$ define $C_{i}(h)=\mathcal{R}\left(h, \partial \mathbf{R}_{+}^{3}\right) \cap U_{i}$. (F) and (P1) imply that the $C_{i}(h)$ are compact and disjoint. The stable manifold theorem and the long tubular flow theorem (e.g. Proposition 1.1. in Chapter 3 of [22]) imply that there exists a neighborhood $\mathcal{N}_{3} \subseteq \mathcal{N}_{2}$ of $g$ such that every $h \in \mathcal{N}_{3}$ satisfies

(P3) If $C_{i}(g)$ is an attracting (repelling) heteroclinic cycle for $g$ restricted to $\partial \mathbf{R}_{+}^{3}$ and is determined by the points $p_{1} \in \operatorname{int} \mathbf{R}_{+}^{\{\sigma(1)\}}, \ldots, p_{k} \in \operatorname{int} \mathbf{R}_{+}^{\{\sigma(k)\}}$, then $C_{i}(h)$ is an attracting (repelling) heteroclinic cycle for $h$ restricted to $\partial \mathbf{R}_{+}^{3}$ and is determined by some points $q_{1} \in \operatorname{int} \mathbf{R}_{+}^{\{\sigma(1)\}}, \ldots, q_{k} \in \operatorname{int} \mathbf{R}_{+}^{\{\sigma(k)\}}$. (P3) is commonly referred to the "robustness" of the heteroclinic cycle $C_{i}(g)$ (see, e.g., [15] for a review). (P1)-(P3) imply that for any $h \in \mathcal{N}_{3}, C_{i}(h)$ is a connected component of $\mathcal{R}\left(h, \partial \mathbf{R}_{+}^{3}\right)$ and is of the same "type" as $C_{i}(g)$ (e.g., if $C_{i}(h)$ is a periodic orbit, then $C_{i}(g)$ is a periodic orbit). Lemma 5 and the stable manifold theorem imply there exists a neighborhood $\mathcal{N}_{4} \subseteq \mathcal{N}_{3}$ of $g$ such that every $h \in \mathcal{N}_{4}$ satisfies

(P4) Given $S \subseteq\{1,2,3\}, W^{s}\left(h, C_{i}(h)\right) \cap \operatorname{int} \mathbf{R}_{+}^{S} \neq \emptyset$ if and only if $W^{s}\left(g, C_{i}(g)\right)$ $\cap \operatorname{int} \mathbf{R}_{+}^{S} \neq \emptyset$.

(P4) and Theorem 3 imply that for any $S \subseteq\{1,2,3\}$ and $h \in \mathcal{N}_{4}, h_{S}$ is uniformly persistent if and only if $g_{S}$ is uniformly persistent. Hence, we have shown that $g$ has robust communities.

\section{Proof of Theorem 1}

Let $f \in \mathcal{P}_{3}$ be a vector field such that 0 a connected component of $\mathcal{R}\left(f, \partial \mathbf{R}_{+}^{3}\right)$. Let $\mathcal{N} \subseteq \mathcal{P}_{3}$ be a neighborhood of $f$. In the proof of Theorem 2 we have shown that there exists a $g \in \mathcal{N}$ such that $g$ is Kupka-Smale, has robust communities, 0 is a connected component of $\mathcal{R}\left(g, \partial \mathbf{R}_{+}^{3}\right)$ and any heteroclinic cycle $\gamma \subset \partial \mathbf{R}_{+}^{3}$ for $g$ satisfies one of the eigenvalue inequalities of Lemma 5. In addition, Pugh's general density theorem [25] implies that we can choose $g$ such that $\omega(g, x) \subset \operatorname{int} \mathbf{R}_{+}^{3}$ for some $x \in \operatorname{int} \mathbf{R}_{+}^{3}$ if and only if $\operatorname{Per}(g) \cap \operatorname{int} \mathbf{R}_{+}^{3} \neq \emptyset$. Let $L: \partial \mathbf{R}_{+}^{3} \rightarrow \mathbf{R}_{+}$be a smooth Lyapunov function such that $D L(x) g(x)<0$ for all $x \in \partial \mathbf{R}^{3} \backslash \mathcal{R}\left(g, \partial \mathbf{R}_{+}^{3}\right)$. Choose $\delta>0$ such that $(\mathrm{F})$ holds. Let $\mathcal{N}_{4} \subseteq \mathcal{N}$ be a neighborhood of $g$ such that every $h \in \mathcal{N}_{4}$ satisfies (P1)-(P4). Since the elements of $\operatorname{Per}(g)$ are hyperbolic, we can choose a neighborhood $\mathcal{N}_{5} \subseteq \mathcal{N}_{4}$ of $g$ such that every $h \in \mathcal{N}_{5}$ satisfies

(P5) $\omega(h, x) \subset \operatorname{int} \mathbf{R}_{+}^{3}$ for some $x \in \operatorname{int} \mathbf{R}_{+}^{3}$ if and only if $\operatorname{Per}(h) \cap \operatorname{int} \mathbf{R}_{+}^{3} \neq \emptyset$.

To verify that $f$ is successionally stable, it suffices to check two things. First, we need to verify that $S \subseteq\{1,2,3\}$ is a community for $g$ if and only if $S$ is community for every $h \in \mathcal{N}_{5}$. This follows immediately from Theorem 2. Second, given two distinct communities, $S$ and $T$, for $g$ (hence also communities for all $h \in \mathcal{N}_{5}$ ) and $I \subseteq\{1,2,3\} \backslash S$, we need to verify that $S \stackrel{I}{\rightarrow} T$ for $g$ if and only if $S \stackrel{I}{\rightarrow} T$ for every $h$ in a neighborhood of $g$. We break down this verification into three cases. First, suppose that $|S \cup I| \leq 2$. Then (P1)-(P4) imply that $S \stackrel{I}{\rightarrow} T$ for $g$ if and only if $S \stackrel{I}{\rightarrow} T$ for every $h \in \mathcal{N}_{4}$. Second, suppose that $S \cup I=\{1,2,3\}$ and $T=\{1,2,3\}$. 
In this case, $S \stackrel{I}{\rightarrow} T$ occurs for $h$ if and only if $h$ is uniformly persistent. Since $g$ is uniformly persistent if and only if every $h \in \mathcal{N}_{5}$ is uniformly persistent, we have verified this case. Finally, suppose that $S \cup I=\{1,2,3\}$ and $|T| \leq 2$. In this case, $S \stackrel{I}{\rightarrow} T$ occurs for $h \in \mathcal{N}_{5}$ if and only if the following two conditions hold:

(C1) $W^{s}\left(C_{i}(h)\right) \cap \operatorname{int} \mathbf{R}_{+}^{3}=\emptyset$ for all connected components $C_{i}(h)$ of $\mathcal{R}\left(h, \partial \mathbf{R}_{+}^{3} \backslash \operatorname{int} \mathbf{R}_{+}^{T}\right)$.

(C2) $\omega(h, x) \subset \partial \mathbf{R}_{+}^{3}$ for all $x \in \operatorname{int} \mathbf{R}^{3}$.

(P4) implies that (C1) is satisfied for $h \in \mathcal{N}_{5}$ if and only if (C1) is satisfied for $g$. (P5) implies that if (C2) is not satisfied for $g$, then (C2) is not satisfied for any $h \in \mathcal{N}_{5}$. Alternatively suppose that (C2) is satisfied for $g$. Then $L: \partial \mathbf{R}_{+}^{3} \rightarrow \mathbf{R}_{+}$ extends to a Lyapunov function $\tilde{L}: \mathbf{R}_{+}^{3} \rightarrow \mathbf{R}_{+}$such that $D \tilde{L}(x) g(x)<0$ for all $x \in \operatorname{int} \mathbf{R}^{3}$. (P1)-(P4), Lemma 5 and the stable manifold theorem imply that there exist a compact neighborhood $V \subset \mathbf{R}_{+}^{3}$ of $\mathcal{R}\left(g, \partial \mathbf{R}_{+}^{3}\right)$ and a neighborhood $\mathcal{N}_{6} \subseteq \mathcal{N}_{5}$ of $g$ such that every $h \in \mathcal{N}_{6}$ satisfies

(P6) $V$ is an isolating neighborhood for $\mathcal{R}\left(h, \partial \mathbf{R}_{+}^{3}\right)$.

Since $D \tilde{L}(x) g(x)<0$ for all $x \in \mathbf{R}_{+}^{3} \backslash \mathcal{R}\left(g, \partial \mathbf{R}_{+}^{3}\right)$, we can choose a neighborhood $\mathcal{N}_{7} \subseteq \mathcal{N}_{6}$ such that every $h \in \mathcal{N}_{7}$ satisfies

(P7) $D \tilde{L}(x) h(x)<0$ for $x \in \mathbf{R}_{+}^{3} \backslash V$.

(P6) and (P7) imply that if (C2) holds for $g$, then (C2) holds for all $h \in \mathcal{N}_{7}$. Hence $S \stackrel{I}{\rightarrow} T$ for $h \in \mathcal{N}_{7}$ if and only if $S \stackrel{I}{\rightarrow} T$ for $g$.

\section{ACKNOWLEDGMENT}

The author thanks an anonymous referee for comments and corrections which improved the content and exposition of this paper.

\section{REFERENCES}

[1] R. A. Armstrong and R. McGehee, Competitive exclusion, Amer. Natur. 116 (1980), 151-170. MR 82d:92029

[2] W. Brannath, Heteroclinic networks on the tetrahedron, Nonlinearity 7 (1994), 1367-1384. MR 95e:58128

[3] G. J. Butler, H. I. Freedman, and P. Waltman, Uniformly persistent systems, Proc. Amer. Math. Soc. 96 (1986), 425-430. MR 87d:58119

[4] G. J. Butler and P. Waltman, Persistence in dynamical systems, J. Differential Equations 63 (1986), 255-263. MR 87k:54058

[5] T. J. Case, Invasion resistence, species build-up and community collapse in metapopulation models with interspecies competition, Bio. J. of the Linn. Soc. 42 (1991), 239-266.

[6] C. Conley, Isolated Invariant Sets and Morse Index, Amer. Math. Soc., CBMS 38 (1978). MR 80c:58009

[7] J. A. Drake, The mechanics of community assembly and succession, J. Theor. Bio. 147 (1990), 213-233.

[8] B. M. Garay, Uniform persistence and chain recurrence, J. Math. Anal. and Appl. 139 (1989), 372-382. MR 90h:54049

[9] A. Hastings and A. Klebanoff, Chaos in three-species food chains, J. Math. Biol. 32 (1994), 427-451. MR 95g:92019

[10] J. Hofbauer, Heteroclinic cycles in ecological differential equations, Tatra Mt. Math. Publ 4 (1994), 105-116. MR 95i:34083

[11] J. Hofbauer and K. Sigmund, The theory of evolution and dynamical systems: mathematical aspects of selection, Cambridge University Press, Cambridge, 1988. MR 91h:92019 
[12] S. B. Hsu, S. P. Hubbell, and P. Waltman, A mathematical theory for single-nutrient competition in continuous cultures of microorganisms, SIAM J. Appl. Math. 32 (1977), 366-383. MR 55:7458

[13] S. B. Hsu, H. L. Smith, and P. Waltman, Competitive exclusion and coexistence for competitive systems on ordered Banach spaces, Trans. Amer. Math. Soc. 348 (1996), 4083-4094. MR 97d:92021

[14] V. Hutson and K. Schmitt, Permanence and the dynamics of biological systems, Math. Biosci. 111 (1992), 1-71. MR 93d:92003

[15] M. Krupa, Robust heteroclinic cycles, J. Nonlinear Sci. 7 (1997), 129-176. MR 98g:58126

[16] M. Krupa and I. Melbourne, Asymptotic stability of heteroclinic cycles in systems with symmetry, Ergod. Th. \& Dynam. Sys. 15 (1995), 121-147. MR 96g:58101

[17] I. Kupka, Contribution á la theorie des champs generiques, Contrib. Diff. Eqs. 2 (1963), 457-484. MR 29:2818a

[18] R. Law and R. D. Morton, Alternative permanent states of ecological communities, Ecology 74 (1993), 1347-1361.

[19] _ Permanence and the assembly of ecological communities, Ecology 77 (1996), 762775.

[20] R. M. May, Stability and complexity in model ecosystems, 2nd edn., Princeton University Press, Princeton, 1975.

[21] Z. Nitecki and M. Shub, Filtrations, decompositions and explosions, Amer. J. Math. 97 (1975), 1029-1047. MR 52:15561

[22] J. Palis and W. de Melo, Geometric theory of dynamical systems, Springer-Verlag, New York, 1982. MR 84a:58004

[23] M. M. Peixoto, On an approximation theorem of Kupka and Smale, J. Differential Equations 3 (1966), 214-227. MR 35:499

[24] W. M. Post and S. L. Pimm, Community assembly and food web stability, Math. Biosci. 64 (1983), 169-192.

[25] C. Pugh, An improved closing lemma and a general density theorem, Amer. J. Math 89 (1967), 1010-1021. MR 37:2257

[26] S. J. Schreiber, Generalist and specialist predators that mediate permanence in ecological communities, J. Math. Bio. 36 (1997), 133-148. MR 98k:92017

[27] P. Schuster, K. Sigmund, and R. Wolff, Dynamical systems under constant organization 3: Cooperative and competitive behavior of hypercycles, J. Differential Equations 32 (1979), 357-368. MR 82b:34035b

[28] S. Smale, Stable manifolds for differential equations and diffeomorphisms, Ann. Scuola Normale Superiore Pisa 18 (1963), 97-116. MR 29:2818b

[29] H. L. Smith and P. Waltman, Competition for a single limiting resource in continuous culture: the variable-yield model, SIAM J. Appl. Math. 54 (1994), 1113-1131. MR 95b:92014

[30] F. W. Wilson, Smoothing derivatives of functions and applications, Trans. Amer. Math. Soc. 139 (1969), 413-428. MR 40:4974

[31] G. S. K. Wolkowicz, Successful invasion of a food web in a chemostat, Math. Biosci. 93 (1989), 249-268. MR 90f:92027

Department of Mathematics, Western Washington University, Bellingham, WashingTON 98225

E-mail address: sschreib@cc.wwu.edu 